\title{
Research and Modeling of Mechanical Crosstalk in Linear Arrays of Ultrasonic Transducers
}

\author{
Mateusz CELMER, Krzysztof J. OPIELIŃSKI \\ Chair of Acoustics and Multimedia, Faculty of Electronics, \\ Wroctaw University of Science and Technology \\ Wybrzeże Wyspiańskiego 27, 50-370 Wrocław, Poland; \\ e-mail: \{mateusz.celmer, krzysztof.opielinski\}@pwr.edu.pl
}

(received April 11, 2016; accepted July 12, 2016)

\begin{abstract}
Linear arrays of ultrasonic transducers are commonly used as ultrasonic probes in medical diagnostics for imaging the interior of a human body in vivo. The crosstalk phenomenon occurs during the operation of transducers in which electrical voltages and mechanical vibrations are transmitted to adjacent components. As a result of such additional excitation of the transducers in the array, the directivity characteristics of the aperture used changes, and consequently there is interference with proper operation of a given array and the emergence of distortions in the obtained ultrasound image that reduce its quality. This paper studies the manner of propagation of mechanical crosstalk in the designed model of a linear array of ultrasonic transducers on the basis of unwanted signals, which appeared on elementary piezoelectric transducers when power is supplied to the selected transducer in the array. The universal model of linear array of ultrasonic transducers, which has been developed, allowed the simulation of mechanical crosstalk, taking into account the cross-coupling phenomenon in all of its structure with the use of finite elements method (FEM) implemented in COMSOL Multiphysics software. The analysis of crosstalk signals showed that they consist of aggregated pulses propagating with different speeds and frequencies. This signifies the formation of different vibration modes transmitted simultaneously via different paths. The paper is an original approach which enables to identify different vibration modes and estimate their participation in the crosstalk signal and their ways of propagation. Conclusions from the research allow predicting specific design changes which are significant due to the minimization of mechanical crosstalk in linear arrays of ultrasonic transducers.
\end{abstract}

Keywords: mechanical crosstalk; ultrasonic transducer array; FEM modeling; COMSOL Multiphysics.

\section{Introduction}

The intensive development of electronics in recent years allows the use of sophisticated methods and algorithms for multi-channel digital control of advanced processes for acquisition and processing of data in pseudo real-time. This has led to the development of advanced methods for shaping the ultrasound beam - focusing, steering, sweeping - in the area of ultrasonic medical diagnostics and non-destructive testing. Therefore, a market has developed for the production of multi-element arrays of ultrasonic transducers, which enable the multi-channel generation and recording of ultrasonic signals propagated in different media (BERTORA, 2007). Over the past 20 years, there has been a rapid growth in the construction and miniaturization of 1-D, 2-D and even 3-D multi- element arrays of ultrasonic transducers for use in nondestructive testing of materials and in medical diagnostics in vivo (BERTORA, 2007; DRINKWATER, WILCOX, 2006; Eames, Hossack, 2008; Gudra, Opielinski, 2006; Martínez-Graullera et al., 2011; OpielinSKI, 2012; OpIELIŃSKI et al., 2015; WILDES et al., 1997). Currently, one of technologies which are heavily developed is MEMS (MicroElectroMechanical Systems) used, among others, in the fabrication of miniaturized CMUT (Capacitive Micromachined Ultrasonic Transducers) ultrasonic arrays for medical diagnostics and ultrasound therapy (KhURI-YAKUB, Oralkan, 2011; Song et al., 2015; Wong et al., 2010).

The most important thing when designing the arrays of ultrasonic transducers is to match the transducers to effective operation in a given medium. In the applications of medical diagnostics for the imaging of 
tissue structures in vivo, elementary array transducers are powered by pulses and their desirable characteristics are high efficiency, effectiveness, sensitivity, and wide bandwidth for converting electricity to acoustic energy. This allows the imaging of tissue structures at greater depths and obtaining a high axial (along the direction of ultrasound propagation) resolution of imaging. It can be achieved by increase of energy generated by an elementary transducer in the array increasing its effective surface area, however, this limits the lateral resolution of imaging, therefore, in the case of linear arrays, the transducers have the shape of narrow pillars. Divergence of the wave beam in elevation (along the length of transducers) is limited by an acoustic lens. The wide band of elementary transducers in the array can be achieved by the use of piezoceramics with a low acoustic impedance, the use of piezoelectric composite, loading the back transducer surface using the attenuating layer, and matching the acoustic impedance of the transducer to the acoustic impedance of tissue using front matching layers (BERTORA, 2007; NAKAMURA, 2012). It is equally important to ensure the repeatability of parameters of individual elementary transducers in the array and automate the process of its manufacturing.

The fabrication of ultrasound transducer arrays is complex and diverse, and the impact of their design on the parameters of the transducer array is often difficult to predict. This is due to the interaction of vibrating elements, which are close to one another in the array structure (the so-called cross-coupling effect (KINO, BAER, 1983)). Cross-coupling can occur in all structural components of the array (the backing layer, front layers, transducer mounts) as well as in a medium designed for the operation of the array. This phenomenon is caused by overlapping and propagation of different vibration modes (longitudinal, transverse, and surface waves, Lamb waves) in the array and the medium which is adjacent to its surface. Figure 1 shows the example of cross-coupling. In this case, the elementary transducers are designed to work on thickness vibration. Electrical excitation of one component causes thickness vibrations in it that are transferred to the second adjacent element, while the first and the

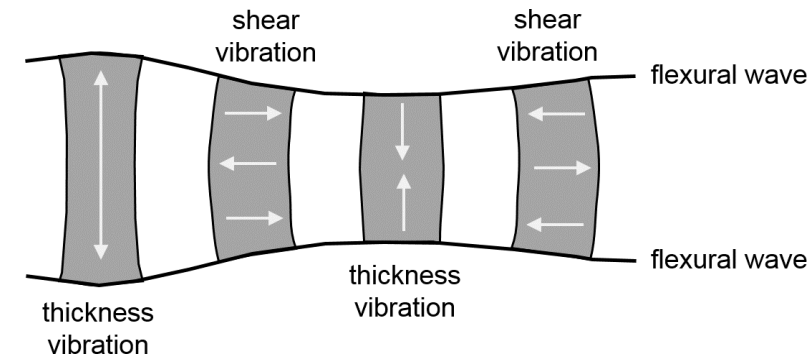

Fig. 1. Schematic example of coupling between the thickness mode and the shear mode in the structure of an array of piezoelectric composite transducers (the cross-section through the thickness of the array) (WILM et al., 2004). third elements are excited to shear vibrations. In addition, the ultrasonic transducers in the array can be excited to vibrate as a result of permeation of electrical voltages between the system of signal electrode and common ground electrode leads.

The result of these phenomena in ultrasonic arrays is the appearance of ultrasonic signals of relatively small amplitude in the elementary piezoelectric transducers which have been excited to operate in an undesirable manner by electrical or mechanical means, resulting in the transmission of signals and vibrations between the elements of the array (CELMER, Opieliński, 2015a; Guess et al., 1995). The reason is insufficient mechanical and electrical insulation between the elements in the array. Crosstalk depends on many factors, such as the type and geometry of the piezoelectric elements, their distribution, the connection of electrodes, the type of connected conductors, shielding effectiveness of leads, and the construction of the matrix (CELMER, OPIELIŃSKI, 2015a; Guess et al., 1995).

Crosstalk is one of the main causes of noise impairing the quality of ultrasound images obtained because it distorts the directivity of the array and can also lead to a poor pulse response (KINO, BAER, 1983). This is because the signal which excites one elementary transducer also excites adjacent transducers, which in the case of mechanical crosstalk generate small acoustic signals with the appropriate delay, which extends the transmitted and received pulses. The problem of crosstalk is particularly important in the case of arrays with a large number of elementary transducers used to precisely steering and focusing the beam for ultrasound imaging (BERG, RøNNEKLEIV, 2006). In this case, many array transducers are simultaneously excited with pulses at different delays. As a result of repeated, uncontrollable mutual crosstalk, which adds up in different phases, an inconsistent sound field is created with undesirable peaks and troughs outside the focus. The design of the transducer array is specific due to the distribution of electrical and mechanical crosstalk. The four main stages of the production of linear arrays used in ultrasound B-mode scanners are shown schematically in Fig. 2 (BERTORA, 2007). Piezo-
1 - piezoceramic glued

to backing (loading) layer

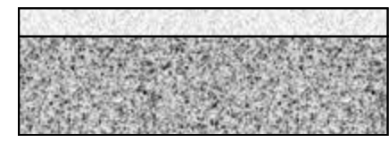

3 - kerf filled

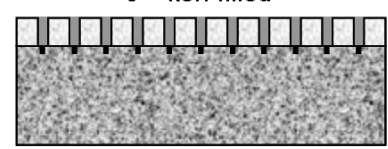

2 - piezoceramic incision

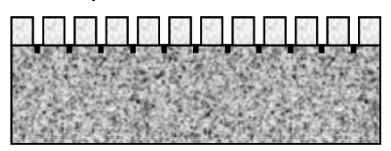

4 - front matching layers applied

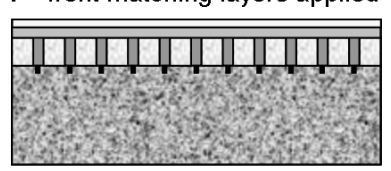

Fig. 2. The main stages of the production of standard linear ultrasonic probes (BERTORA, 2007). 


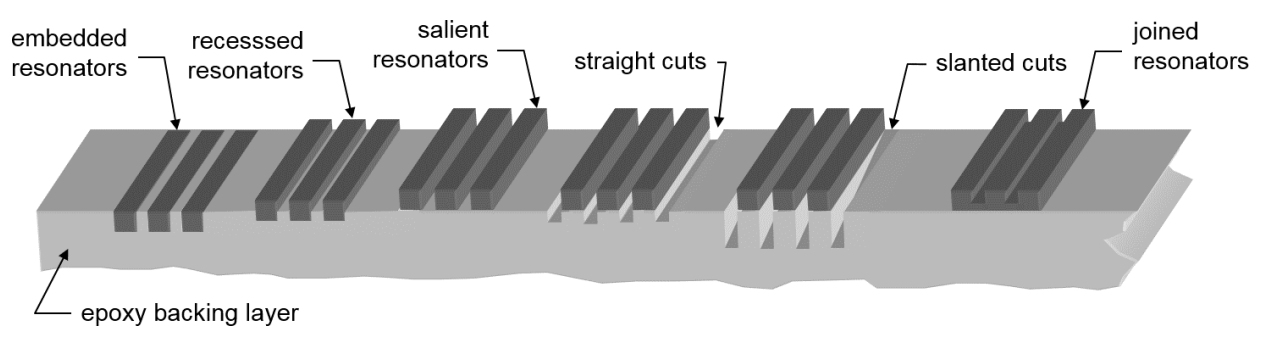

Fig. 3. Example of different saw cut patterns of piezoceramics with backing layer.

electric ceramics is glued to the back loading layer and cut in order to separate elementary transducers in the array. The cuts (kerfs) are filled with a suitable material, and usually 1 or 2 front matching layers and the elevation-focusing lens are applied on top.

Next, the signal wires are attached to individual piezoelectric elements and the assembly is moulded in a plastic block and encased to produce the finished ultrasonic probe (BERTORA, 2007). Most often, however, it is required that the acoustic coupling factor of backing layer is close to one in order to obtain the smallest mechanical quality factor of transducers in the array, therefore the transducers are filled from behind with the layer of epoxy resin mixed with tungsten powder or glass microbaloons. Good coupling in turn leads to more efficient transfer of mechanical vibrations through the back layer. In order to prevent this, one can make deeper kerfs between the transducers (Fig. 3), which in turn will increase the lateral deflections of the resulting protruding fragments (KINO, BAER, 1983); kerfs may also be shallow enough that piezoceramics remains connected (continuous) at the base. Type of material for filling the kerfs also affects the vibration characteristics of the whole system.

Mechanical crosstalk is also affected by imposing continuous front matching layers on already cut transducers or before cutting them. One should note that these small thickness (quarter wavelength) layers must be applied with great accuracy and precision. A specific design of arrays reduces or improves the connection of electrodes. Electrodes of transducers in arrays manufactured according to the method shown in Fig. 2 (metallization on the radiating and back surface) are often connected to the paths etched on a flexible thin film. Cutting the transducers on the back layer is easy from the point of view of production automation, but causes uncontrolled changes of parameters of individual elements after cutting, which should show the best possible repeatability.

Cutting piezoceramics to elementary transducer plates before merging with the back layer allows the automatic measurements of frequency admittance characteristics, determination of electromechanical parameters and making precise selections for their repeatability with the assumed margin of dispersion. The most important thing is to maintain the repeatability of resonant frequency of thickness vibrations and the electrical conductance in resonance, which determines the repeatable efficiency and sensitivity of transducers (OpIELIŃsKi et al., 2010a; 2010b). Cutting transducers at a preliminary stage of production is also necessary in the case of arrays with a curved surface (GUDRA, OPIELINSKI, 2006; OpIELIŃsKI et al., 2015). Figure 4 shows the image of cut piezoceramics used to build ultrasonic arrays.

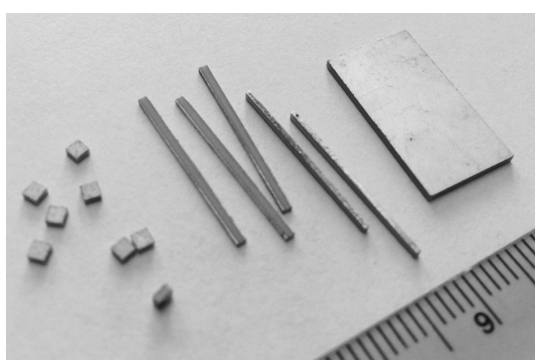

Fig. 4. Piezoceramic plates used to build ultrasonic arrays.

Cutting the piezoceramic plates at the initial stage of manufacturing allows the selection in terms of parameter repeatability, but hinders the precise positioning of the adjacent plates with a predetermined small gap. This gap along with the wide of the transducer (pitch) should be not more than a half of a wavelength, whereby there are no side lobes in directional characteristics within the angle of $90^{\circ}$. In order to precisely position the elements and easily connect the leads to the electrodes, a pair of rigid PCBs is used with suitably etched paths and grooves to support the transducers in the rear, near the edges of their length. PCBs affect the cross-coupling phenomenon, because they are in a direct mechanical contact with the vibrating transducers. This way of support, however, limits the transverse vibrations in the width of the transducers. In order to facilitate soldering of paths which supply excitation voltages to the electrodes and the ground paths, specific etching of the part of the surface of electrodes in piezoceramic plates is often made, which also affects the crosstalk and electro-mechanical parameters of transducers in the array. Figure 5 schematically shows the two discussed ways to design a linear array of piezoceramic transducers with and without supporting the elementary transducers (BERTORA, 2007; Celmer, Opieliński, 2015a). 
a)

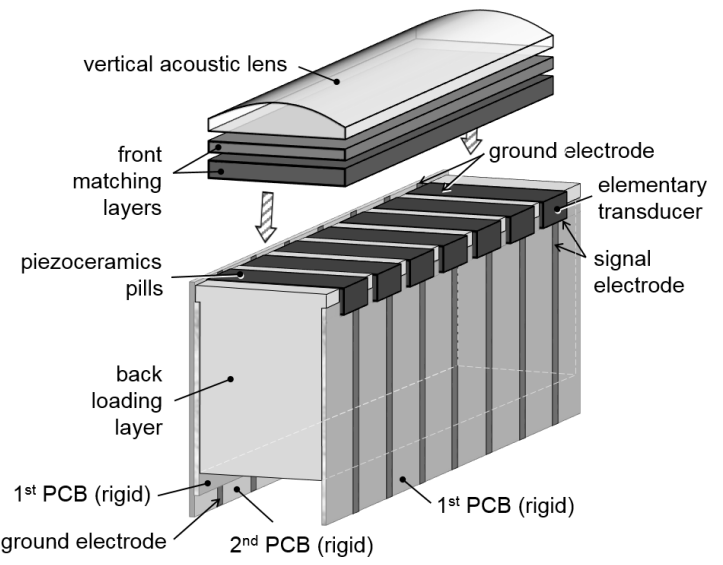

b)

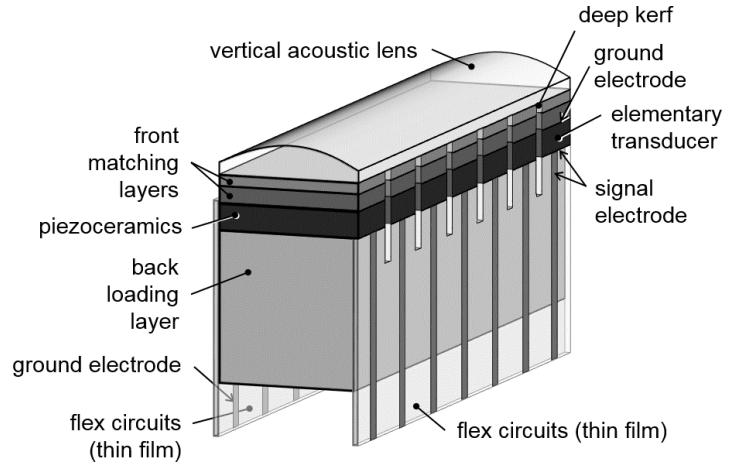

Fig. 5. The diagram of the design of a linear array of piezoceramic transducers with (a) and without (b) supporting the elementary transducers.

As it has been demonstrated, all design elements and their mutual positioning and integration have a significant impact on the distribution of mechanical vibrations and electrical voltages in the array and its parameters on one hand, and the complexity and cost of manufacturing on the other hand, which should be reasonably optimized. The arrays of ultrasonic transducers have responses which do not conform to simple theories; the problem has been very difficult to approach theoretically, therefore, the array designs are often verified experimentally and modified at various stages of construction. It seems reasonable to use the finite elements method (FEM) implemented in commercial software. There are different theoretical approaches and different models used, which are more or less complex but most of them require multi-day calculations using supercomputers. It also lacks effective methods of a fast analysis of calculation results to identify paths of the crosstalk propagation in the standard linear arrays used in B-mode ultrasound scanners. The aim of this paper is to create a model of such a standard array structure, which could be further developed after the initial experimental verification, taking into account the increasingly complex designs, and use that model to determine the mechanical crosstalk and their causes and effects using COMSOL Multiphysics ${ }^{\circledR}$ software (COMSOL, 2012). This in effect should help to design or fast modify ultrasonic arrays in order to reduce the mechanical crosstalk. The novelty of this work is to propose the fast and effective method of the FEM calculated crosstalk signal analysis by the average of speed propagation values in the context of maximum amplitudes and levels of characteristic peaks of the crosstalk spectrum. Such an analysis is possible thanks to the originally-developed virtual model of linear ultrasonic array consisting of simple structural elements that can be progressively complemented to obtain the complete construction. This approach allows recognizing the routes of crosstalk propagation in the form of various modes of vibrations. It has been found that the mechanical contact of the front and rear surface of transducers in the standard linear array is the main cause of crosstalk.

\section{Theory}

Relatively simple and well-known one-dimensional Mason or KLM model can be used to develop the model of a piezoelectric transducer (Nakamura, 2012). These models, however, have a number of limitations that do not allow the simulation of mechanical interactions in the structure of transducer array. In order to model the layout of piezoelectric transducers operating in a particular medium, it is necessary to formulate the governing equations for the piezoelectric device and wave propagation in the acoustic medium. However, it is very complicated to create a consistent analytical models in this case (SHERrit, MukHERJEe, 2012; WóJCIK, 1998), because the equations governing both the piezoelectric effect and acoustic wave propagation involve partial differential equations (PDE). Direct analysis of such a system can be very difficult, especially if the structure is complex. One of the possible numerical techniques that can be used to find the approximate solutions for PDE is FEM, which can solve physical systems of immense complexity through approximations of the PDEs and discretization of the system geometry. It can provide a 3 -D model of the realistic transducer array simulation and a way to visualize the real acoustic wave propagation into the acoustic medium, as well as a simulation of an interaction of the acoustic field of an ultrasonic transducer with other objects.

The FEM is based on the division of areas into finite elements (polygons with 3 or 4 corners) for which the solution is approximated by specific functions, and performing the actual calculations only for nodes (in each element corner) of this division. The advantages of the FEM usage in that case are: 3-D pressure field investigation, modeling of back reflections from any interface, simulation of all vibration modes, consideration of losses in the materials, and simulation of different mechanical and acoustical interactions (Guo, 
Cawley, 1990). Besides the above advantages, finite element modeling has certain disadvantages as well. In order to obtain required accuracy at high frequencies, the finite element model of an ultrasonic transducer comprises several thousands to few million degrees of freedom. Such a large model requires computer systems with extensive processing capabilities and considerable effort to build, debug and operate. Another disadvantage of finite element modeling is that the response of a transducer can differ substantially from the actual response due to the use of an inadequate number of elements to resolve acoustic waves, unrealistic boundary conditions or less accurate model parameters (e.g. material properties). These disadvantages indicate the need for experimental validation of a finite element model.

Nowadays, there are some commercially available packages (such as COMSOL, ANSYS, ABAQUS, PZFLEX, ATILA etc.) for solving complex problems using FEM. However, a modeler or designer needs to know various aspects involved in the finite element modeling to accurately model and analyze a problem. Many authors (including (ABBOUd et al., 1998; Guo, Cawley, 1990; Gutierrez et al., 2010; LerCh, 1990; Medina et al., 2006; Powell et al., 1997) etc.) adopted FEM to analyze ultrasonic transducers. The innovative approach in this paper is the finite element modeling of mechanical crosstalk in the designs of linear periodic arrays of ultrasonic transducers used in medical diagnostics consisting of simple structural elements that can be progressively complemented to obtain the complete construction. This enables to determine which structural elements of the arrays, in which setup and to what extent affect the crosstalk using the specific way of crosstalk signal analysis. In terms of this work the simulation was made in the advanced COMSOL Multiphysics ${ }^{\circledR}$ commercial software, in AcousticPiezoelectric Interaction (Transient). There is a relatively small number of publications related to simulation of crosstalk in ultrasonic arrays using FEM, and the older ones present their own complex mathematical models, and only later ones use advanced, commercially available software packages (ABBOUD et al., 1998; ACEVEDO et al., 2015; BAYRAM et al., 2006; Bybi et al., 2013; Domínguez, Contla, 2013; LAMBERTI, 1999; WILM et al., 2004; ZHOU et al., 2003).
Respective authors have different approaches and specific structures and models of arrays. Only a few publications in recent years use COMSOL software for this purpose. This reflects the novelty of the subject.

The Acoustic-Piezoelectric Interaction, Transient user interface of COMSOL software combines Pressure Acoustics, Transient, Solid Mechanics, Electrostatics, and the Piezoelectric Devices interface features (COMSOL, 2012; 2013). In the pressure acoustics physics interface is the pressure, $p$, the only dependent variable and the governing PDE is formulated as the inhomogeneous Helmholtz equation, used in COMSOL (Blackstock, 2000; COMSOL, 2012; 2013):

$$
\nabla \cdot\left(-\frac{1}{\rho}\left(\nabla p-\mathbf{q}_{d}\right)\right)-\frac{\omega^{2} p}{\rho c^{2}}=Q_{m}
$$

where $p(\mathbf{x}, t)=p(\mathbf{x}) e^{i \omega t}$ is the acoustic pressure in the medium, $\rho$ is the density of the acoustic medium, $c$ is the speed of sound and $\mathbf{q}_{d}\left[\mathrm{~N} / \mathrm{m}^{3}\right]$ and $Q_{m}\left[\mathrm{~s}^{-2}\right]$ are dipole and monopole sources (VIGGEN, 2013), respectively. Typical boundary conditions for the Helmholtz equation (1) are: sound-hard boundaries (walls), sound-soft boundaries, impedance and radiation boundary conditions.

\section{Array models}

This work includes the simulation of mechanical crosstalk in a linear array of piezoelectric transducers using COMSOL for three different and simple models (Fig. 6):

1) Complete (back layer + piezoceramics + front layer + water) with the support of the transducers in the rear using PCB mounting plates with grooves (Fig. 6a),

2) Complete without supporting the transducers (Fig. 6b),

3) Incomplete (without front and back layers) with supporting the transducers (Fig. 6c).

This combination of simulations allows the assessment of the effect of individual construction components on the propagation of mechanical crosstalk for a reasonable calculation time.
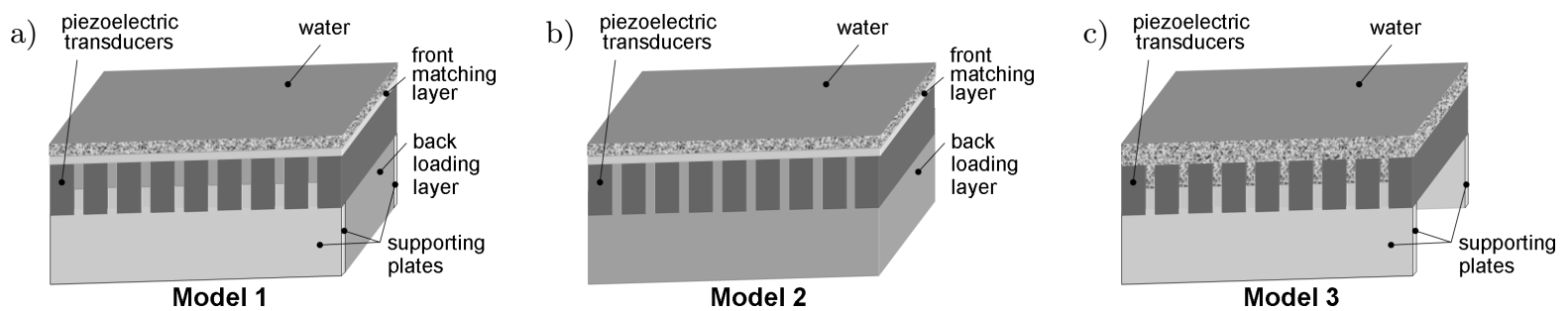

Fig. 6. The developed models of the array of piezoelectric transducers to simulate the crosstalk in COMSOL software: a) complete with supporting transducers, b) complete without supporting transducers, c) incomplete, with supporting transducers. 
The maximum element size of mesh polygon (COMSOL, 2012; 2013):

$$
h_{\max }=\frac{\lambda}{N},
$$

was set for $N=5$, when meshing the models ( $\lambda$ is the ultrasonic wavelength). Because all elements in the constructed mesh are smaller than $h_{\max }$, the limit is set larger than the actual required element size. The simulation was conducted using the frequency of thickness vibration of transducers $f_{r}=2 \mathrm{MHz}$. Selected materials of individual components of the array models are characterized in Table 1.

Table 1. Characteristics of materials of individual components of the array models.

\begin{tabular}{|c|c|c|c|c|}
\hline Material & 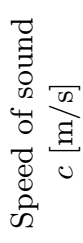 & 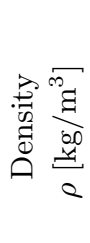 & 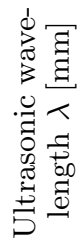 & 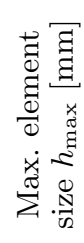 \\
\hline $\begin{array}{l}\text { Piezoceramic: } \\
\text { Noliac NCE51* }\end{array}$ & 4560 & 7850 & 2.28 & 0.456 \\
\hline $\begin{array}{l}\text { Supporting plates: } \\
\text { FR4 Circuit Board }\end{array}$ & 3200 & 1900 & 1.6 & 0.32 \\
\hline $\begin{array}{l}\text { Back layer: filled } \\
\text { epoxy resin (X238) }\end{array}$ & 2650 & 1673 & 1.325 & 0.265 \\
\hline $\begin{array}{l}\text { Matching layer: } \\
\text { polyurethane }\end{array}$ & 1650 & 1250 & 0.825 & 0.165 \\
\hline $\begin{array}{l}\text { Acoustic medium: } \\
\text { Water at } T=20^{\circ} \mathrm{C}\end{array}$ & 1485 & 998 & 0.74 & 0.148 \\
\hline
\end{tabular}

* (Noliac: Piezoceramics specifications. www.noliac.com)

When solving transient acoustic problems where the wave shape is not necessarily harmonic, it may be necessary to resolve its spatial variations with a fine mesh, say with a minimal scale $\mathrm{d} x=h_{\max }$. In order for the numerical solution of the temporal development of the acoustic field to limit acoustic disturbances, it is necessary to restrict the maximal time steps $\mathrm{d} t=\Delta t_{\max }$ taken by the solver. The condition is known as the CFL (Courant-Friedrichs-Lewy) condition (COMSOL, 2013). For transient acoustic problems it is defined as:

$$
C F L=c \cdot \frac{\mathrm{d} t}{\mathrm{~d} x},
$$

where $c$ is the sound speed. This way, the maximal time steps can be expressed by the formula:

$$
\Delta t_{\max }=\frac{C F L \cdot h_{\max }}{c}=\frac{C F L}{N \cdot f_{\max }} .
$$

For applications where all shape functions are quadratic, the Courant-Friedrichs-Lewy number should be around 0.2. This condition restricts any acoustic disturbances to propagate more than $20 \%$ of the mesh size $\mathrm{d} x$ during one time step $\mathrm{d} t$.

Figure 7a shows a complete model of the linear array of piezoelectric transducers with support used for calculations (compare with Fig. 6a), which was meshing in COMSOL, Fig. 7b shows the signal powering the central transducer, and Fig. 7c shows its spectrum.

a)

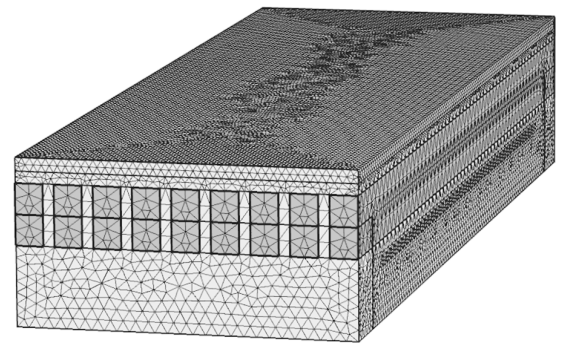

b)

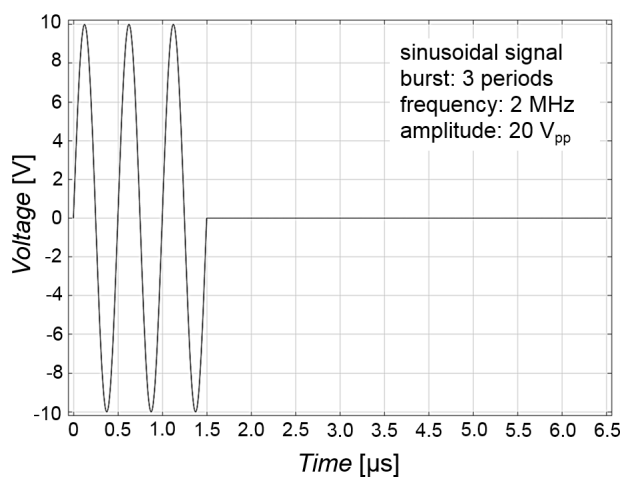

c)

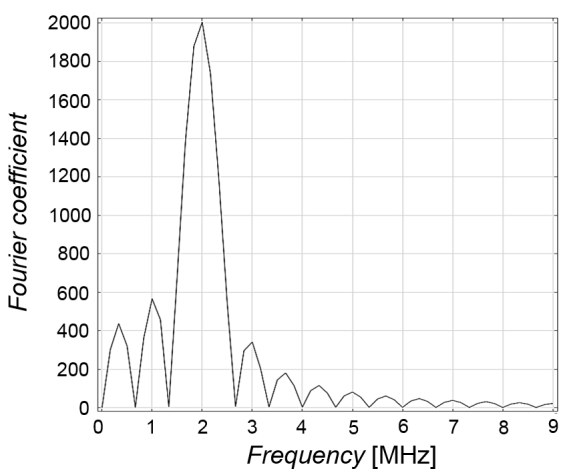

Fig. 7. The complete model of the linear array of piezoelectric transducers with support, meshing in COMSOL for calculations (a), the signal powering the central transducer (b), the spectrum of the powering signal (c).

The assumed thickness $h_{t h}$ of piezoceramic transducers results from the assumed frequency of thickness vibration $f_{r}$ and equals:

$$
h_{t h}=\frac{N_{t h}}{f_{r}}=0.97 \mathrm{~mm},
$$

where $N_{t h}$ is a frequency constant of NCE51 piezoceramics. The assumed width and length of transducers are respectively $0.5 \mathrm{~mm}$ and $18 \mathrm{~mm}$, and the gap 
between them is set at $0.2 \mathrm{~mm}$ (i.e.: $0.7 \mathrm{~mm}$ pitch). The thickness of the front matching layer is equal to a quarter wavelength $(0.20625 \mathrm{~mm})$. The thickness of the back loading layer is $1.515 \mathrm{~mm}$, the thickness of the walls of supporting PCB plates is $1 \mathrm{~mm}$, and the thickness of the water layer is $0.30875 \mathrm{~mm}$ in Model 1 and 2, and $0.515 \mathrm{~mm}$ in Model 3. This way, the height of each of the models together with the water layer is $3 \mathrm{~mm}$. Small layer thickness was assumed in order to shorten the time of calculations. To avoid the effect of multiple reflections in a thin layer of water and a back layer of the models (Fig. 7), non-reflecting boundary condition was set for their external borders, which allows an outgoing wave to leave the medium with minimum reflections (COMSOL, 2013).

\section{Results of calculations and analysis}

The results of the simulation of mechanical crosstalk performed using COMSOL for three developed models of the linear array of piezoelectric transducers (Fig. 6) are shown in Figs. 8-10 as signals appearing on transducers adjacent to the powered transducer and sequentially more and more distant ones (No. 1, 2, 3, 4), as well as the spectra of these signals.
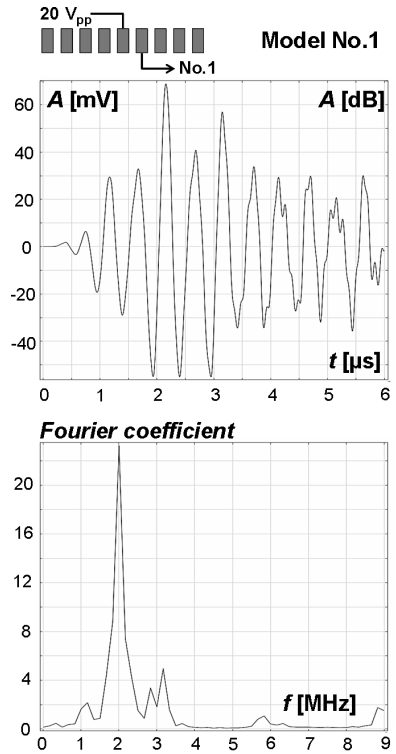

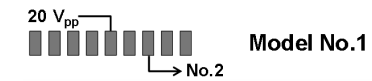
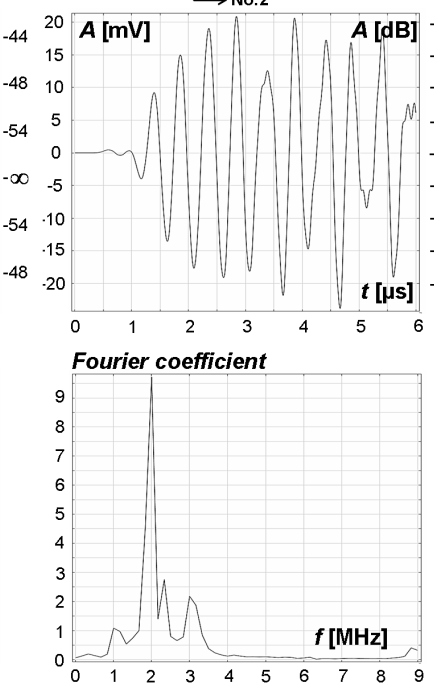

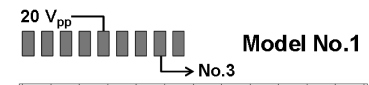
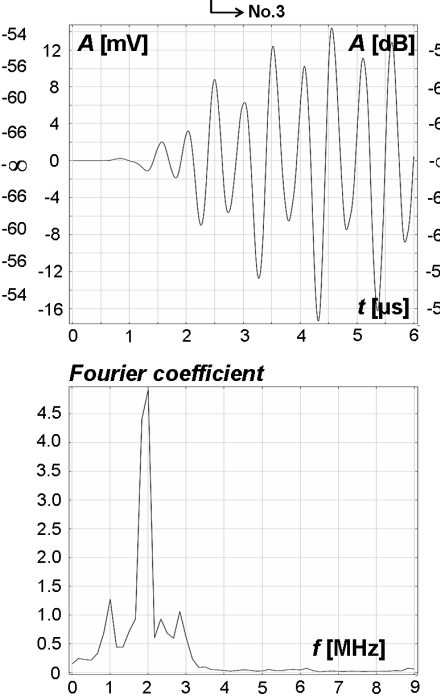
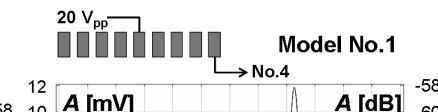

Fig. 8. The results of simulation of mechanical crosstalk in Model No. 1 of the linear array of ultrasonic transducers

(waveforms and spectra).
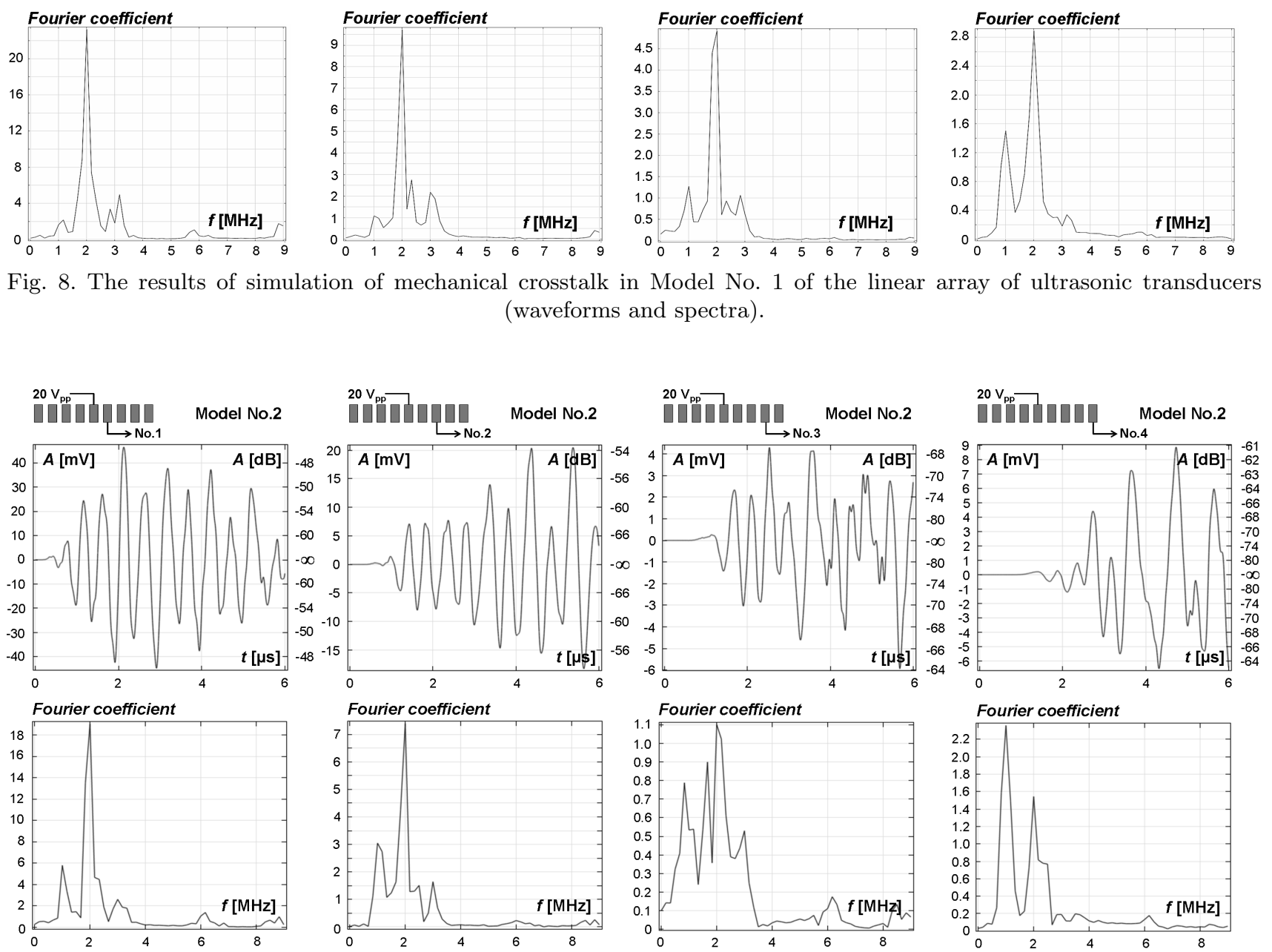

Fig. 9. The results of simulation of mechanical crosstalk in Model No. 2 of the linear array of ultrasonic transducers (waveforms and spectra). 

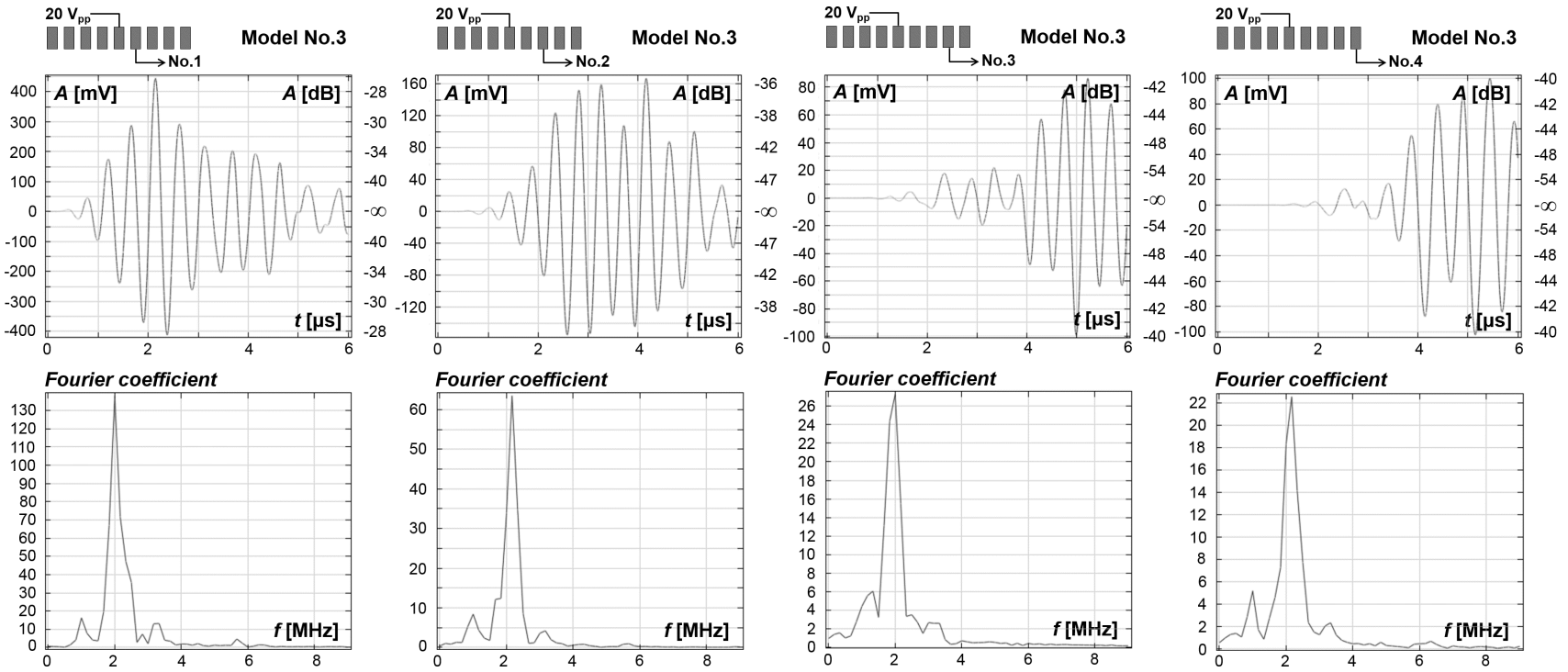

Fig. 10. The results of simulation of mechanical crosstalk in Model No. 3 of the linear array of ultrasonic transducers (waveforms and spectra).

Graphical comparative analysis of the parameters of simulated crosstalk signals and their spectra for individual transducers in the developed models of arrays is shown in Figs. 11-14.
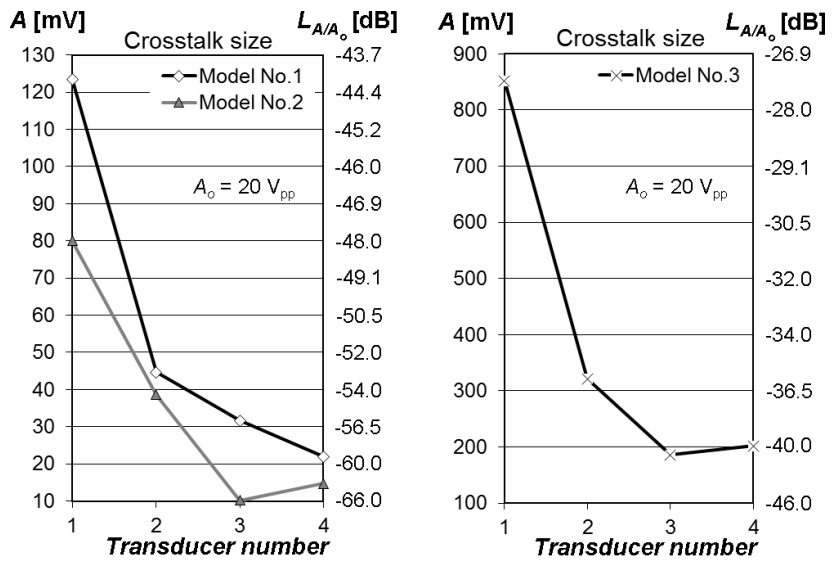

Fig. 11. The maximum peak-to-peak amplitude of the mechanical crosstalk signal $A[\mathrm{mV}]$ ( $Y$ axis on the left) and its level $L_{A / A_{o}}[\mathrm{~dB}]$ with respect to the supply voltage $A_{o}=20 \mathrm{~V}_{p p}$ of the active transducer ( $Y$ axis on the right); values determined from the waveforms of crosstalk signal

(Figs. 8-10).

The analysis of waveform of crosstalk signal and their spectra (Figs. 8-10) confirms the occurrence of cross-coupling in structural components of array models (Fig. 1). Crosstalk signals consist of aggregated pulses propagating with different speeds and frequencies. This signifies the formation of different vibration modes (thickness vibrations, shear vibrations, flexural waves) transmitted simultaneously via different routes (multipath effect in construction). This section includes original approach which enables to identify them and estimate their participation in the crosstalk signal.

The analysis of direct maximum peak-to-peak amplitude values of the mechanical crosstalk signal on the individual transducers No. 1-4 in the array with respect to the activated transducer (No. 0) shows that crosstalk signals are the largest in Model 3, and the smallest in Model 2, and their decay on transducers located farther from the activated transducer is not always monotonic (Fig. 11). The absolute amount of crosstalk may, however, depend on the efficiency of transducers in a given array design (model), so one needs to additionally analyze the distribution of levels of maximum amplitude of spectrum for the crosstalk signal at a resonant frequency $f_{r}=2 \mathrm{MHz}$ with respect to the amplitude of the powering signal spectrum (Fig. 14 - dashed lines). The levels decrease in similar manner in Models 1 and 3, and their values in Model 3 are approximately $16 \mathrm{~dB}$ greater than in Model 1. This means that, as expected, the transducers in Model 3 are more efficient than transducers in Models 1 and 2, hence the crosstalk generated here are bigger. This is caused by the lack of the back layer in Model 3, which reduces back vibrations of transducers in Models 1 and 2. In addition, crosstalk on individual transducers in Model 2 are decaying faster than in Models 1 and 3 (Fig. 14 - dashed lines), which is related to a lower stiffness of the structure in Model 2 (no PCBs supporting the transducers).

The decay in total mechanical crosstalk signal is not monotonic due to their complexity - the aggregation with different phases of different vibration modes with different propagation speeds and frequencies. One can attempt to isolate these modes by analyzing the delay of mechanical crosstalk signal and its visible com- 
ponents (Fig. 12, Figs. 8-10), as well as by means of spectral analysis (Fig. 13, Fig. 14). a)

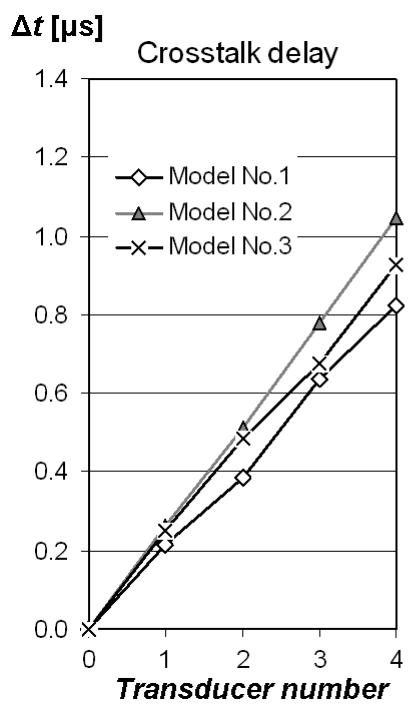

b)

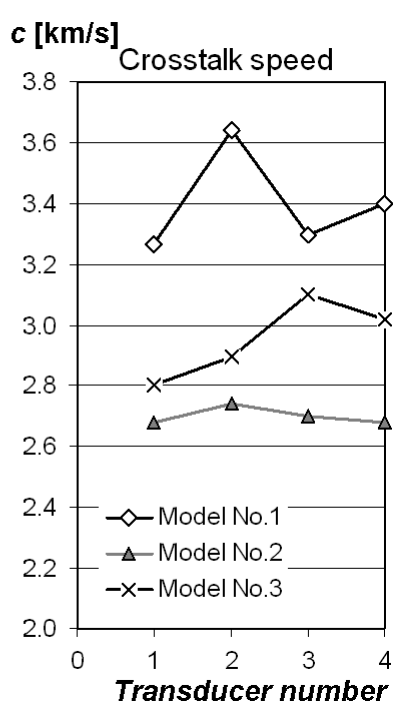

Fig. 12. The delay of mechanical crosstalk signal (a) - the values were determined directly from the waveform of the crosstalk signal (Figs. 8-10) based on the detection of the beginning of the signal; the average speed of propagation of the mechanical crosstalk signal (b) - the values were determined based on the delays in the increasing paths from the center of the activated transducer to the centers of transducers $n=1,2,3,4$, sequentially adjacent $\left(c_{n}=\Delta t_{n} /\left(n \cdot d_{p}\right)\right.$, where pitch $\left.d_{p}=0.7 \mathrm{~mm}\right)$.

The delay of mechanical crosstalk signal on individual transducers $1-4$ in the array relative to the activated transducer (Fig. 12a) increases in all models in a more or less non-linear manner while Model 1 has the smallest delays, and Model 2 - the largest delays. The average speeds of propagation of crosstalk signal in the developed models, determining in sequence from the delays on the increasing paths from the center of the activated transducer to the centers of sequentially adjacent transducers show significant variation (Fig. 12b).

The average speed of propagation in the transducer immediately adjacent to the activated one in Model 1 is about $3200 \mathrm{~m} / \mathrm{s}$, and increases to about $3700 \mathrm{~m} / \mathrm{s}$ on the second one, drops to $3300 \mathrm{~m} / \mathrm{s}$ on a third one further increases to $3400 \mathrm{~m} / \mathrm{s}$ on a fourth one. Analyzing these speeds with respect to the waveforms in Fig. 8 and the speed of ultrasound in the individual structural elements of the array (Table 1 ), we can conclude that in Model 1, crosstalk are carried by the PCB boards supporting the transducers from the rear and thereby stimulated to vibrations. However, these are superimposed by the prevailing crosstalk propagated in the back layer. The increase in the speed of crosstalk on the transducer No. 2 is caused by small lateral vibrations of the activated transducer, propagated by the filling in the gap between the transducers. In this case, the ultrasound wave travels the section in the piezoceramics along half the width of the activated transducer $(0.25 \mathrm{~mm})$, then the gap between the transducers in the epoxy resin $(0.2 \mathrm{~mm})$, the width of transducer No. $1(0.5 \mathrm{~mm})$, another gap in the epoxy resin $(0.2 \mathrm{~mm})$ and the half width of the transducer No. 2 $(0.25 \mathrm{~mm})$. The time of this transition is about $0.34 \mu \mathrm{s}$, which gives the value of about $3700 \mathrm{~m} / \mathrm{s}$ of the average speed of propagation in the width center of transducer No. 2. This type of crosstalk is visible as a small pulse just before the vibration pulse propagated in PCB (Fig. 8 - second waveform from the left). We can assume that a similar lateral wave does not pass through the PCB protrusions (transducer mounts), otherwise the pulses of the shortest runtimes $\left(c_{a v} \approx 4000 \mathrm{~m} / \mathrm{s}\right)$ would appear in the crosstalk signal. These protrusions block the transverse vibrations in the volume of piezoceramics in the recess. Thickness vibrations of the activated transducer trigger a small flexural wave, which stimulates adjacent transducers in the array to thickness and transverse vibrations in varying proportion (Fig. 1) and thus also carry crosstalk. Flexural wave is visible in the form of the modulation of the crosstalk signal (Fig. 8 - all waveforms). Due to the speed of propagation less than the speed of ultrasound in water, flexural vibrations are incorporated into the crosstalk signal. Slow longitudinal waves transmitted in the back, front, and water layer (Table 1) have a small share in the crosstalk signal here and are not visible in the waveforms (Fig. 8).

The average speed of propagation for all transducers in Model 2 is about 2650 to $2750 \mathrm{~m} / \mathrm{s}$ (Fig. 12b), which proves the transmission of these crosstalk signals mainly by the back loading layer (Table 1). Larger values in this range appear in transducers 3 and 4 (Fig. 12b) due to minor crosstalk resulting from transverse vibrations of transducers (Fig. 9 - a small pulses at the beginning of two last waveforms). Pulses of transverse vibrations are small enough in this case that one cannot precisely determine the beginning of the signal, and therefore the speed of crosstalk on transducers 3 and 4 increases only slightly and does not reach the value of $3700 \mathrm{~m} / \mathrm{s}$, as in Model 1. Flexural vibrations in Model 2 also exist - the modulation of crosstalk signal is clearly visible (Fig. 9 - all waveforms). Slow longitudinal waves transmitted in the back, front, and water layer (Tale 1) have a small share in the crosstalk signal here and are not visible in the waveforms (Fig. 9).

In Model 3, which lacks the front and rear layers, the propagation of thickness vibrations prevails in the water layer adjacent to the radiating surface of the transducers in the array (Fig. 10, Fig. 12b) at the speed of $1485 \mathrm{~m} / \mathrm{s}$ (Table 1 ), however, they comprise vibrations of a small amplitude propagated as the result of the transmission of the wave in PCBs $(c=3200 \mathrm{~m} / \mathrm{s})$ and even smaller transverse vibrations 
of transducers propagated through the water filling the gaps between them $(c \approx 2800 \mathrm{~m} / \mathrm{s})$. We can assume that a similar lateral wave does not pass through the PCB protrusions (transducer mounts), otherwise the pulses of the shortest runtimes $\left(c_{a v} \approx 4000 \mathrm{~m} / \mathrm{s}\right)$ would appear in the crosstalk signal. Flexural vibrations are also transmitted in the construction - the modulation of crosstalk signal is visible (Fig. 10 - all waveforms). Slow longitudinal waves transmitted in the front water layer (Table 1) have a prevailing share in the crosstalk signal here.

The results of the foregoing analysis of mechanical crosstalk propagation pathways in the designs of the developed models of linear arrays of ultrasound transducers are shown schematically in Fig. 15.

The frequency of vibration modes that make up the crosstalk signals can be determined by analyzing the characteristic components of the spectra of these signals (Figs. 8-10). The results of this analysis are shown in Fig. 13 and Fig. 14. Vibrations transmitted in crosstalk signals in each of array models comprise three frequencies: $1 \mathrm{MHz}, 2 \mathrm{MHz}, 3 \mathrm{MHz}$, whose energy share in the spectra are specific to each model, however, the share of the resonant frequency $f_{r}=2 \mathrm{MHz}$ is always the biggest (Fig. 13).

Based on the calculations and analyzes conducted in this paper, we can assume that the resonant component $f_{r}=2 \mathrm{MHz}$ consists of waves propagated as the result of the transmission of thickness vibrations of the piezoceramic transducer in the form of longitudinal ultrasound waves passing with the respective speeds (Table 1) directly through the back layer, PCBs, front layer and water layer near the surface of the array (Fig. 15). The component $f=3 \mathrm{MHz}$ consists of waves propagated as the result of the transmission of transverse vibrations of the piezoceramic transducer in
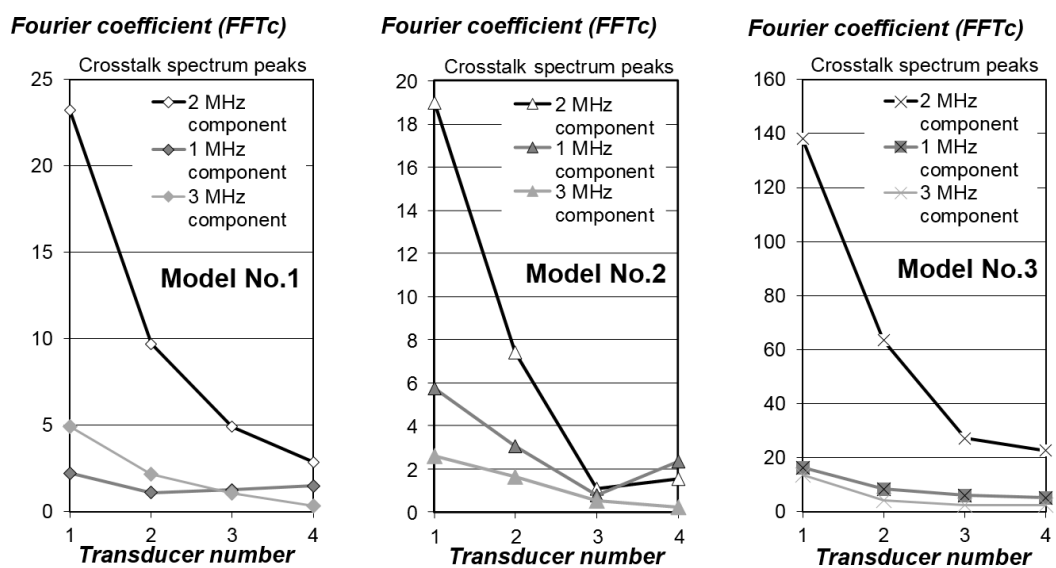

Fig. 13. The maximum amplitudes of characteristic peaks of the spectrum of the crosstalk signal (components for $f=1 \mathrm{MHz}, 2 \mathrm{MHz}, 3 \mathrm{MHz}$ ) - values determined directly from the spectra of the crosstalk signal (Figs. 8-10).
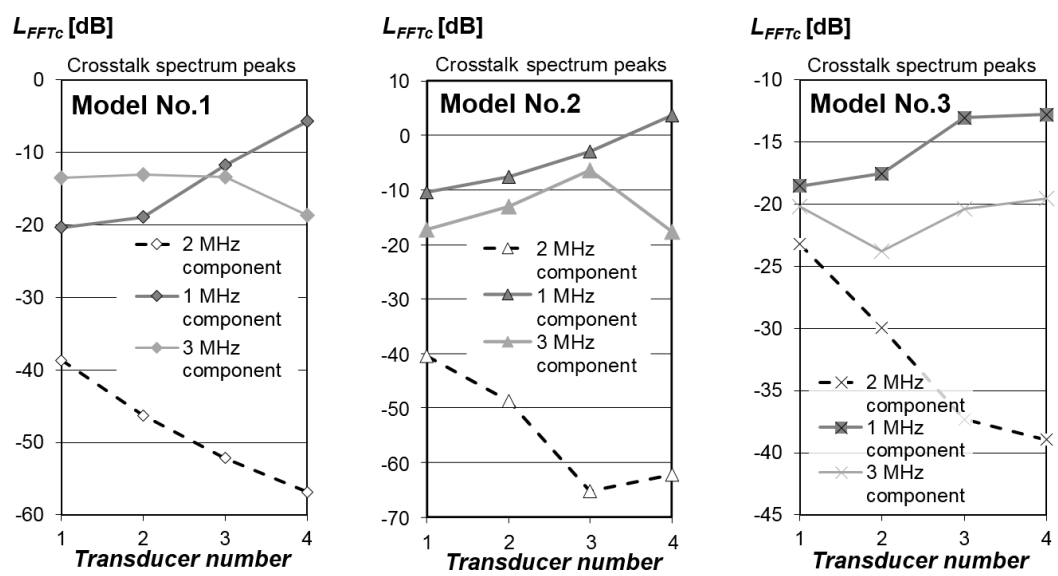

Fig. 14. The maximum amplitude levels of characteristic peaks of the spectrum of the crosstalk signal (components for $f=1 \mathrm{MHz}, 2 \mathrm{MHZ}, 3 \mathrm{MHz}$ ) - values determined directly from Fig. 13, wherein the level of component $f=2 \mathrm{MHz}$ (dashed line) is referred to the amplitude of this component in the spectrum of the powering signal (Fig. 7c), and the level of other components is referred to the respective amplitudes of the component $f=2 \mathrm{MHz}$ in the same spectra from Fig. 13. 


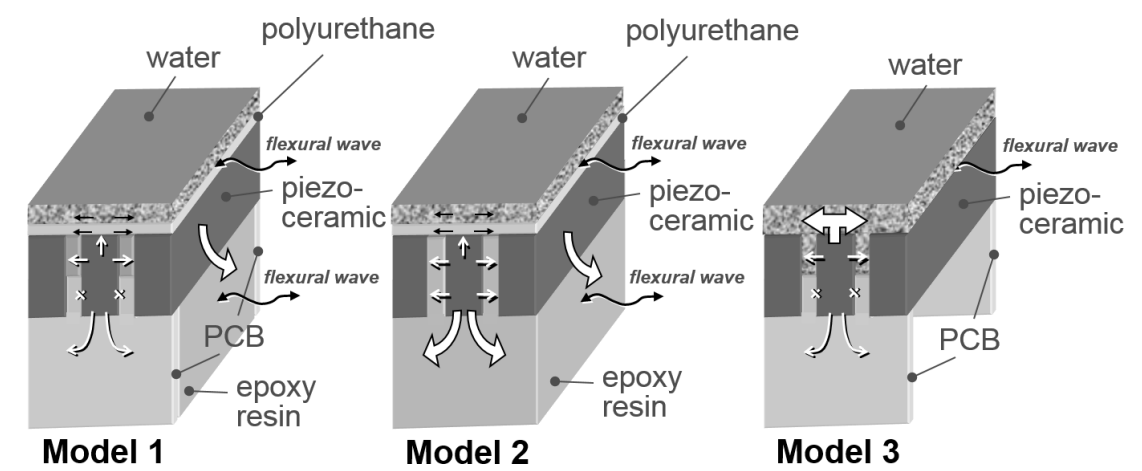

Fig. 15. Schematic representation of mechanical crosstalk propagation pathways in the designs of the developed models of linear arrays of ultrasound transducers.

the form of longitudinal ultrasound waves passing with the respective speeds (Table 1 ) through piezoceramics fragments and the materials filling the gaps between them in the array (Fig. 15). The frequency of about $3 \mathrm{MHz}$ is due the frequency constant of NCE51 piezoceramics for transverse vibrations (Noliac: Piezoceramics specifications). The component $f=1 \mathrm{MHz}$ consists of waves propagated as the result of transformation of thickness vibrations of the piezoceramic transducer to flexural waves propagating at low speed throughout the whole structure of the linear array, along its length (Fig. 15).

The lowest amplitude values of the spectrum (Fourier coefficient $-F F T c$ ) in the case of component $2 \mathrm{MHz}$ exist for Model 2, they are slightly larger for Model 1 and definitely large for Model 3 (Fig. 13). However, in the case of Model 3, it involves the increased efficiency, as noted earlier. Note that Model 3 was designed in order to verify the distribution of crosstalk after removing the back and matching layers, what is not exercised in the design of arrays intended for pulse operation, because in this case, with a significant increase in the quality factor of transducers, the narrowing of the operating bandwidth and extension of the duration of the generated pulses in media (tissue, water) take place at the expense of the increased efficiency. Transverse vibrations of transducers are the most attenuated in Model 2 and a little less in Model 1 (Fig. 13). In turn, flexural vibrations are the smallest in Model 1 and slightly larger in Model 2 (Fig. 13), which can be explained by the greater rigidity of the array in Model 1 along its length due to the use of PCBs.

Figure 14 shows the share of individual frequency components of the mechanical crosstalk signal in analyzed models of arrays. The level of component $f=$ $2 \mathrm{MHz}$ (dashed line) is referred to the amplitude of this component in the spectrum of the powering signal (Fig. 7c), and the level of other components (solid lines) is referred to the respective amplitudes of the component $f=2 \mathrm{MHz}$ in the same spectra from Figs. 8-10 and 13 .
Amplitude levels of the crosstalk spectrum of the resonant component $(2 \mathrm{MHz})$ are the lowest in Model 2, slightly higher in Model 1 and the largest in Model 3 (resulting from a high efficiency due to the absence of the back layer). Moreover, this decreases in subsequent transducers, the fastest in Model 2, and the slowest in Model 3.

The level of $3 \mathrm{MHz}$ component (transversal vibrations of the transducer) is the greatest in Model 1 $(-13 \mathrm{~dB})$ is maintained at transducers Nos. $1-3$ and is reduced to about $-20 \mathrm{~dB}$ at the transducer No. 4. In Model 2, the level of this component is about $-17 \mathrm{~dB}$ on the transducer No. 1 and slightly increases at the transducer No. $2(-13 \mathrm{~dB})$, then more increases to $-6 \mathrm{~dB}$ on the transducer No. 3, returning to the level of $-17 \mathrm{~dB}$ on the transducer No. 4. By analyzing those changes with respect to Model 1, we can conclude that the stiffening of transducers on the edges in the recesses of PCBs reduces the distribution of transverse vibrations in the piezoceramics. On the other hand, the level of the component responsible for the distribution of transverse vibrations changes in the range of -20 to $-24 \mathrm{~dB}$ in Model 3 for similar reasons as in Model 1.

The levels of flexural vibrations component (1 $\mathrm{MHz}$ ) are the smallest in Model 1, slightly larger in Model 3 and the largest in Model 2 (higher by about $10 \mathrm{~dB}$ than in Model 1). In general, there is a tendency of the increase in subsequent transducers, which means the increase in flexural vibration amplitude in the array line while moving away from the central transducer.

\section{Conclusions}

The universal model of linear array of ultrasonic transducers, which has been developed, allows the simulation of mechanical crosstalk, taking into account the cross-coupling phenomenon in all of its structure with the use of finite elements method (FEM) using the inhomogeneous Helmholtz equation implemented in COMSOL Multiphysics ${ }^{\circledR}$ software. This model is simple and takes into account the most important 
presently used conventional designs of linear arrays, while allowing their elementary division with a resolution of no less than $1 / 5$ wavelength of ultrasound. This provides a relatively fast calculation time and the capability to delete, replace, and add individual structures in order to investigate their effect on the distribution of mechanical crosstalk in the array.

Mechanical crosstalks in the linear array of ultrasonic transducers were simulated for three specific versions of the designed model in the form of signals appearing on transducers adjacent to the activated transducer, and then they were examined and analyzed, which made it possible to assess the impact of individual components on the size and ways of propagation of the crosstalk in the array. This analysis allowed the separation of characteristic vibration modes, determine their parameters and ways of propagation. It has been found that the mechanical contact of the front and rear surface of transducers in the array, which undergoes thickness vibrations, with layers immediately adjacent thereto, so that these vibrations are transmitted within the structure of these layers as a longitudinal ultrasonic wave with a resonance frequency, is the main cause of crosstalk. The formation of a flexural wave (with a frequency equal to a half the resonant frequency) propagated in continuous layers along the transducer array is also the reason for mechanical crosstalk, although much smaller. Transverse transducer vibrations at a frequency greater than the resonant one and small amplitudes also propagate in the structure of the array due to the width of piezoceramic pillars which does not differ significantly from their thickness (two times less). These vibrations depend on the method of attachment and support of pillars. They are added to the crosstalk signal, and are transmitted along the array of transducers due to the mechanical contact of vibrating side surfaces of transducers with the material which fills the gaps between them. The share of the individual vibration modes in the crosstalk signal depends on the design of the array and the distance between the tested and activated transducer; for the nearest 2-3 transducers, the resonance mode usually plays the largest part.

It is very difficult to simulate crosstalk signals on the shape and the nature of exactly consistent with measurements due to the calculation complexity, model simplifications and boundary conditions but taking into account the measurements of mechanical crosstalk in linear ultrasound arrays shown in the literature (BALlandras et al., 2000; Domínguez et al., 2011; Guess et al., 1995; KIno, BAER, 1983; LAMBERTI, 1999; ZHOU et al., 2003) and obtained by the authors (CElmer, Opieliński, 2014; Celmer, Opieliński, 2015a; Celmer, Opieliński, 2015b; OPIELIŃSKI et al., 2014), simulations carried out in this paper can be considered reliable. The obtained calculation results confirmed that mechanical crosstalk ef- fects occur on adjacent transducers of the array, with a certain delay, as pulses, the amplitude of which fast decreases, in relation to the signal activating a transmitting transducer, with longer distance from that transducer. Longer distance causes the increase of the average delay. The detailed, experimental verification of the results obtained here will be carried out in future research, because it requires building the arrays which reflect the designs of the developed models in at least 3 versions.

Conclusions from the research allow predicting specific design changes which are significant due to the minimization of mechanical crosstalk in linear arrays of ultrasonic transducers. We can limit the distribution of resonant mode crosstalk in PCBs (which mount the piezoceramic pillars) by dispersing the ultrasonic wave on the discontinuities introduced in the structure of PCB plates (by drilling holes, kerfing the surface). The same wave in the back layer can be attenuated, e.g., by doping the epoxy resin with corundum powder or air-filled glass microbaloons, which will increase the density and ultrasound attenuation coefficient but will not substantially change the speed of ultrasound in the resin. The propagation of transverse vibrations in piezoceramics can be reduced by using composite transducers, as well as by cutting gaps between the transducers so that they contain air. Another way to limit the propagation of transducer transverse vibrations can be grinding the side surfaces of piezoceramic pillars in such a way that they are not parallel to each other. The flexural wave propagation can be limited by stiffening the structure of the array along its length, introducing more matching layers and introducing discontinuities to long layers and structural components conveying this type of vibrations. The set of three array models designed in this paper for FEM calculations allows the introduction of the foregoing design changes and simulation of mechanical crosstalk in the array in order to check which ones might prove to be most effective in practice, which is planned in subsequent research.

\section{References}

1. Abboud N.N., Wojcik G.L., Vaughan D.K., Mould J., Powell D.J., Nikodym L. (1998), Finite element modeling for ultrasonic transducers, Medical Imaging: Ultrasonic Transducer Engineering, Proc. SPIE, 3341, 19-42.

2. Acevedo P., García-Nocetti D.F., Recuero M., SÁNCHEZ I. (2015), Simulation of the crosstalk effect of a piezoelectric matrix array oscillating in the lateral mode, International Journal of Physical Sciences, 3, 2, 021-031.

3. Ballandras S., Edoa P.F., Langrognet F., SteIChen W., Pierre G. (2000), Prediction and measurement of cross-talk effects in a periodic linear array built 
using ultrasound micromachining, Proceedings of the IEEE Ultrasonics Symposium, San Juan, Puerto Rico, 1139-1142.

4. Bayram B., Yaralioglu G.G., Kupnik M., KhuriYAKuB B.T., GinZTON E.L. (2006), Acoustic crosstalk reduction method for CMUT arrays, IEEE Ultrasonics Symposium, 590-593.

5. Berg S., Rønnekleiv A. (2006), Reducing fluid coupled crosstalk between membranes in CMUT arrays by introducing a lossy top layer, 2006 IEEE Ultrasonic Symposium, 594-597.

6. Bertora F. (2007), Ultrasound transducers, [in:] Physics for Medical Imaging Applications, Ch. 1, Y. Lemoigne et al. [Eds.], Springer, 111-121.

7. Blackstock D.T. (2000), Fundamentals of Physical Acoustics, John Wiley and Sons, Inc., 568 pages.

8. Bybi A., Assaad J., Hladky-Hennion A.-Ch., BenMeddour F., Grondel S., Rivart F. (2013), Numerical study of the cross-talk effects in acoustical transducer arrays and correction, Proceedings of Meetings on Acoustics, 19 (030049), 8 pages.

9. Celmer M., Opieliński K.J. (2014), Crosstalk effects in multielement ultrasonic transducer arrays, European Acoustics Association Proceedings of the 7th Forum Acusticum, Krakow, Poland, 5 pages.

10. Celmer M., Opieliński K.J. (2015), Study of Crosstalk in Linear Ultrasonic Transducer Arrays, Acta Acustica united with Acustica, 101, 46-54.

11. Celmer M., Opieliński K.J. (2015), Research on crosstalk in commercial ultrasonography arrays [in Polish], [in:] Progress of Acoustics, K.J. Opieliński [Ed.], Wrocław Division of Polish Acoustical Society, Wrocław, 63-74.

12. COMSOL (2012), Comsol Multiphysics User's Guide, ver. 4.3, COMSOL Multiphisics ${ }^{\circledR}$, www.comsol.com, 1292 pages.

13. COMSOL (2013), Acoustic Module User's Guide, ver.4.3b, COMSOL Multiphisics ${ }^{\circledR}$, www.comsol.com, 444 pages.

14. Dominguez I.S., Contla P.A., Hernandez E.M., von KRuger M.A. (2011), Crosstalk effects caused by the geometry of piezoelectric elements in matrix ultrasonic transducers, Brazilian Journal of Biomedical Engineering, 27, 90-97.

15. Domínguez I.S., Contla P.A. (2013), Construction and Characterization of an Ultrasonic Array Using Different Backing Materials to Evaluate Crosstalk, Journal of Materials Science and Engineering B, 3, 8, 493497.

16. Drinkwater B.W., Wilcox P.D. (2006), Ultrasonic arrays for nondestructive evaluation: A review, NDT \& E International, 39, 525-541.

17. EAmes M.D.C., Hossack J.A. (2008), Fabrication and evaluation of fully-sampled, twodimensional transducer array for "Sonic Window" imaging system, Ultrasonics, 48, 376-383.
18. Gudra T., Opielinski K.J. (2006), The ultrasonic probe for the investigating of internal object structure by ultrasound transmission tomography, Ultrasonics, 44, e679-e683.

19. Guess F., OAKley' C.C., Douglas S.J., MorGAN R.D. (1995), Cross-talk paths in array transducers, Proceedings of the IEEE Ultrasonics Symposium, 1279-1282.

20. Guo N.Q., Cawley P. (1990), Three dimensional analysis of the vibration characteristics of piezoelectric discs, Review of Progress in Quantitative Nondestructive Evaluation, 9, 789-794.

21. Gutierrez M.I., Vera A., Leija L. (2010), Finite element modeling of acoustic field of physiotherapy ultrasonic transducers and the comparison with measurements, [in:] Pan American Health Care Exchange (PAHCE), 76-80.

22. Khuri-Yakub B.T., Oralkan Ö. (2011), Capacitive micromachined ultrasonic transducers for medical imaging and therapy, Journal of Micromechanics and Microengineering, 21, 5, 54004-54014.

23. Kino G., BAer R. (1983), Theory for cross-coupling, 1983 IEEE Ultrasonics Symposium, 2, 1013-1019.

24. LAmberti N. (1999), Radiation pattern distortion caused by the interelement coupling in linear array transducer, IEEE Ultrasonics Symposium, 1071-1075.

25. LERCH R. (1990), Simulation of piezoelectric devices by two- and three-dimensional finite elements, IEEE transactions on Ultrasonics, Ferroelectric and Frequency Control, 37, 2, 233-247.

26. Martínez-Graullera O., Gomez-Ullate L., Romero D., Martín C.J., Godoy G. (2011), Design of Curvilinear Array Apertures for 3D Ultrasonic Imaging, [in:] Ultrasound Imaging, Ch. 2, Masayuki Tanabe [Ed.], INTECH, Rijeka, 17-36.

27. Medina J.E.S.M., Buiochi F., Adamowski J.C. (2006), Numerical modeling of a circular piezoelectric ultrasonic transducer radiating in water, ABCM Symposium Series in Mechatronics, 2, 458-464.

28. NAKAmURA K. (2012), Ultrasonic Transducers. Materials and Design for Sensors, Actuators and Medical Applications, Woodhead Publishing Limited, 722 pages.

29. Noliac: Piezoceramics specifications. www.noliac.com.

30. Opieliński K.J., Gudra T., Pruchnicki P. (2010), A digitally controlled model of an active ultrasonic transducer matrix for projection imaging of biological media, Archives of Acoustics, 35, 1, 75-90.

31. Opieliński K.J., Gudra T., Pruchnicki P. (2010), Narrow beam ultrasonic transducer matrix model for projection imaging of biological media, Archives of Acoustics, 35, 1, 91-109.

32. Opielinski K.J. (2012), Ultrasonic Projection, [in:] Ultrasonic Waves, Ch. 1, A. Dos Santos Junior [Ed.], INTECH, Rijeka, 29-58. 
33. Opieliński K.J., Celmer M., Pruchnicki P., Roguski W., Gudra T., Majewski J., Bulkowski M., Piotrowski T., Wiktorowicz A. (2014), The effect of crosstalk in a circular transducer array on ultrasound transmission tomography of breast, Proceedings of Meetings on Acoustics, 21, 167th Meeting of the Acoustical Society of America, Providence, Rhode Island, 075001, 6 pages.

34. Opieliński K.J., Pruchnicki P., Gudra T., Podgórski P., Kurcz J., KraŚnicki T., SĄSiaDEK M., MAJEWSKI J. (2015), Imaging results of multi-modal ultrasound computerized tomography system designed for breast diagnosis, Computerized Medical Imaging and Graphics, 46, 83-94.

35. Powell D.J., Wojcik G.L., Desilets C.S., Gururaja T.R., Guggenberger K., Sherrit S., MukHERJEE B.K. (1997), Incremental "Model-Build-Test" validation exercise for a 1-D biomedical ultrasonic imaging array, IEEE Ultrasonics Symposium, 2, 16691674.

36. Sherrit S., Mukherjee B.K. (2012), Characterization of Piezoelectric Materials for Transducers, [in:] Dielectric and Ferroelectric Reviews, Srowthi S.N., Bharadwaja and Robert A. Dorey[Eds.], Research Singpost, 175-244.

37. Song J., Xue Ch., He Ch., Zhang R., Mu L., Cui J., Miao J., Liu Y., Zhang W. (2015), Capacitive Micromachined Ultrasonic Transducers (CMUTs) for Underwater Imaging Applications, Sensors, 15, 23205-23217.

38. Viggen E.M. (2013), Acoustic multipole sources from the Boltzmann equation, 36th Scandinavian Symposium on Physical Acoustics, 5 pages.

39. Wildes D.G., Chiao R.Y., Daft C.M.W., RigBy K.W., Smith L.S., Thomenius K.E. (1997), Elevation performance of $1.25 D$ and $1.5 D$ transducer arrays, IEEE Transactions on Ultrasonics, Ferroelectrics, and Frequency Control, 44, 1027-1037.

40. Wilm M., Armati R., Daniau W., Ballandras S. (2004), Cross-talk phenomena in a 1-3 connectivity piezoelectric composite, JASA, 116, 5, 2948-2955.

41. Wong S.H., Kupnik M., Watkins R.D., ButtsPauly K., Khuri-Yakub B.T. (2010), Capacitive Micromachined Ultrasonic Transducers for Therapeutic Ultrasound Applications, IEEE Transactions on Biomedical Engineering, 57, 1, 114-123.

42. WóJCIK J. (1998), Conservation of energy and absorption in acoustic fields for linear and nonlinear propagation, Journal of Acoustical Society of America, 104, 5, 2654-2663.

43. Zhou S., Wojcik G.L., Hossack J.A. (2003), An Approach for Reducing Adjacent Element Crosstalk in Ultrasound Arrays, IEEE Transactions on Ultrasonics, Ferroelectrics, and Frequency Control, 50, 12, 17521761. 